\title{
Atomic Scale Structure and Chemical Composition across Order-Disorder Interfaces
}

\author{
R. Srinivasan, R. Banerjee, ${ }^{2}$ J. Y. Hwang, ${ }^{2}$ G. B. Viswanathan, ${ }^{1}$ J. Tiley, ${ }^{3}$ D. M. Dimiduk, ${ }^{3}$ and H. L. Fraser ${ }^{1}$ \\ ${ }^{1}$ Center for the Accelerated Maturation of Materials, Department of Materials Science and Engineering, The Ohio State University, \\ Columbus, Ohio 43210, USA \\ ${ }^{2}$ Center for Advanced Research and Technology, Department of Materials Science and Engineering, University of North Texas, \\ Denton, Texas 76203, USA \\ ${ }^{3}$ Materials and Manufacturing Directorate, Air Force Research Laboratory, Dayton, Ohio 45309, USA
}

(Received 19 November 2008; published 25 February 2009)

\begin{abstract}
Through a combination of aberration-corrected high-resolution scanning transmission electron microscopy and three-dimensional atom probe tomography, the true atomic-scale structure and change in chemical composition across the complex order-disorder interface in a metallic alloy has been determined. The study reveals the presence of two interfacial widths, one corresponding to an order-disorder transition, and the other to the compositional transition across the interface, raising fundamental questions regarding the definition of the interfacial width in such systems.
\end{abstract}

PACS numbers: 68.35.- p, 68.37.Ma, 68.37.Vj

Interfaces play an important role in determining several properties in multiphase systems [1-3]. Atomic-scale renditions of the interface structure have been primarily accomplished through high-resolution transmission electron microscope (HRTEM) phase-contrast images (and simulations) that allow for identification of the positions of atomic columns and structural defects at the interface [4,5]. Recent advances in HRTEM and HRSTEM have been enabled by the development of spherical aberration correctors for the electron probe as well as the image [6-8]. In parallel, developments in first-principles based electronic structure calculations and larger scale atomistic modeling tools permit more accurate simulations of the interface structure $[9,10]$. Recent atomistic simulations of the interface between an ordered precipitate and disordered matrix in a nickel-base superalloy indicate that the interface is not abrupt at the atomic scale [9]. While the implications of these results on the microstructural stability [11-13] and high temperature mechanical properties [1419] are substantial, there is no clear experimental evidence confirming these atomistic simulations. The limitations associated with the resolution of experimental techniques have so far prevented direct atomic-scale imaging and interpretation of the structural and compositional transition across such interfaces. Techniques such as 3D Atom Probe (3DAP) Tomography have enabled detailed exploration of nanometer-scale elemental partitioning across interphase interfaces, including the $\gamma / \gamma^{\prime}$ interface in nickel base alloys [20-26]. In addition, developments in aberrationcorrected HRSTEM now permit $Z$ contrast imaging (arising from the differences in atomic numbers) and interpretation at atomic resolution [27-29]. Our study provides direct experimental evidence at the atomic scale of the true structure and composition of the $\gamma / \gamma^{\prime}$ interface in a nickel base alloy by coupling two advanced and complementary techniques: aberration-corrected HRSTEM and 3DAP.
This work was carried out on the nickel base superalloy Rene' 88 DT [nominal composition $55.63 \mathrm{Ni}-18.02 \mathrm{Cr}$ $13.00 \mathrm{Co}-4.74 \mathrm{Ti}-4.45 \mathrm{Al}-2.48 \mathrm{Mo}-1.21 \mathrm{~W}-0.46 \mathrm{Nb}$ (in at \%)], with superior creep and fatigue properties for turbine disk applications [14-16]. The typical microstructure consists of a disordered fcc $\gamma$ matrix with ordered $L 1_{2} \gamma^{\prime}$ precipitates of varying sizes depending upon processing history [16]. Bore and rim specimens from a turbine disk [30] were subjected to a supersolvus anneal $\left(30 \mathrm{~min}\right.$. at $\left.1150{ }^{\circ} \mathrm{C}\right)$, in the single $\gamma$ phase field, followed by cooling at $24^{\circ} \mathrm{C} / \mathrm{min}$ to room temperature, and subsequent aging for $50 \mathrm{~h}$ at $760{ }^{\circ} \mathrm{C}$ (to achieve a near-equilibrium condition), resulting in the formation of larger $(>200 \mathrm{~nm})$ primary $\gamma^{\prime}$ and smaller $(<50 \mathrm{~nm})$ secondary $\gamma^{\prime}$ precipitates [see Energy Filtered TEM (EFTEM) image in Fig. 1(a)]. Samples for EFTEM and HRSTEM studies were prepared by machining of $3 \mathrm{~mm}$ discs, followed by $\mathrm{Ar}^{+}$ion milling on Fischione Model 1010 and Model 1040 systems. EFTEM studies were carried out using the $\mathrm{Cr} M$ edge (at $42 \mathrm{eV}$ ) in the electron energy loss spectrum to enhance contrast between the Cr-depleted $\gamma^{\prime}$ precipitates and the $\mathrm{Cr}$-enriched $\gamma$ matrix [31].

Atomic resolution, $Z$ contrast, imaging [through High Angle Annular Dark Field (HAADF)-HRSTEM] was performed on a FEI Titan 80-300 microscope, operated at $300 \mathrm{kV}$, equipped with a CEOS probe aberration corrector. Primary $\gamma^{\prime}$ precipitates were chosen to ensure a planar interface parallel to the beam direction, across the foil thickness. To ensure consistency between the HRSTEM and 3DAP analyses, a $\gamma / \gamma^{\prime}$ interface parallel to the $\{002\}$ planes was specifically chosen. The atomic structure of such an interface is shown in the HAADF-HRSTEM image in Fig. 1(b) (note the intensity maxima at spatial frequencies that correspond to the $\{100\}$ and $\{110\}$ superlattice reflections from the $\gamma^{\prime}$ in the Fourier transform). A filtered section of the HRSTEM image, with improved image contrast, is shown in Fig. 1(c). Note that while all atomic 


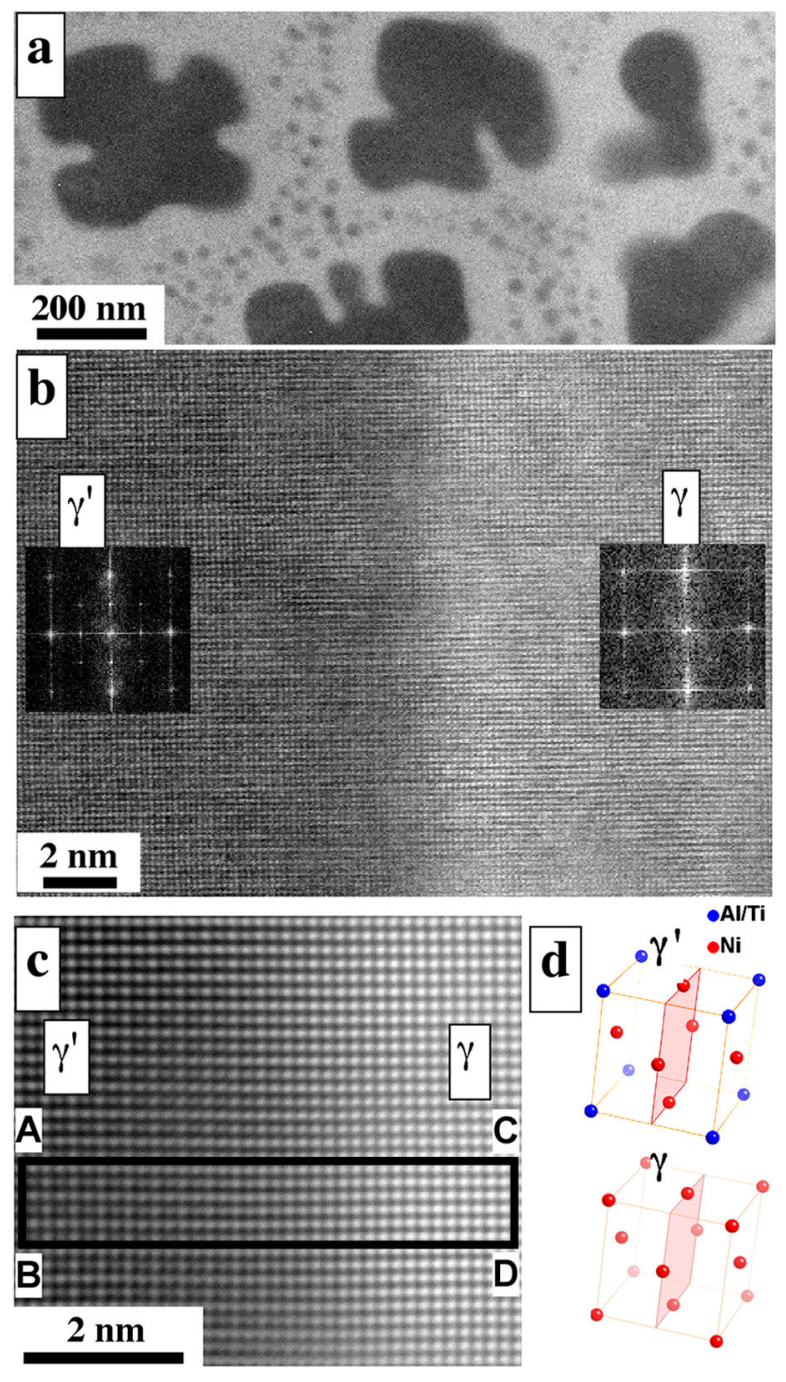

FIG. 1 (color online). (a) Cr-M edge EFTEM low magnification image showing the typical microstructure in Rene88DT slow cooled from $1150{ }^{\circ} \mathrm{C}$ (at $30^{\circ} \mathrm{C} / \mathrm{min}$ ) and aged $50 \mathrm{~h}$ at $760{ }^{\circ} \mathrm{C}$; the larger particles represent the primary $\gamma^{\prime}$ while the smaller particles represent the secondary $\gamma^{\prime}$, (b) HRSTEM image along the [100] zone showing the interface between a primary $\gamma^{\prime}$ precipitate and $\gamma$ matrix; the insets show Fourier Transforms indicating order within the $\gamma^{\prime}$ and lack of order within the $\gamma$ phase, (c) higher magnification filtered HRSTEM image showing the structural and compositional transition across the $\gamma / \gamma^{\prime}$ interface, (d) schematic diagram showing the $\gamma^{\prime}$ and $\gamma$ unit cells, with the (002) plane indicated; alternating (002) rows of $\mathrm{Ni}$ and $(\mathrm{Ni}+\mathrm{Al} / \mathrm{Ti})$ atoms in the $\gamma^{\prime}$ can be visualized, while no such order exists in the $\gamma$ phase.

columns exhibit similar intensities in the $\gamma$, alternating brighter and darker rows of atomic columns (representative of $\{002\}$ planes) are observed in the $\gamma^{\prime}$, due to differences in the average atomic numbers of individual atomic columns. The ordered $L 1_{2}, \mathrm{Ni}_{3} \mathrm{Al}$-based, structure of the $\gamma^{\prime}$ phase consists of two distinct sublattices, the Ni sublattice corresponding to the face-center positions and the $\mathrm{Al}$ sublattice corresponding to the corner positions of the cubic unit cell. This yields alternating $\{002\}$ planes of pure $\mathrm{Ni}$ and $50 \% \mathrm{Ni}$ and $50 \% \mathrm{Al}$ atoms, respectively, when viewed along the $\langle 100\rangle$ direction [Fig. 1(d)]. In case of Rene'88 DT (with several alloying elements), the $\gamma^{\prime}$ phase exhibits alternating heavier Ni-rich (with other substitutional atoms such as $\mathrm{Co}$ ) and lighter Al-rich (with other atoms such as Ti) $\{002\}$ planes [refer left half of Figs. 1(b) and 1(c)], presenting direct evidence of the $L 1_{2}$ superlattice structure. To probe the compositional and structural transition from disordered $\gamma$ to ordered $\gamma^{\prime}$, an intensity profile is plotted across the interface by averaging over the box $A B C D$ [from Fig. 1(c)], as shown in Fig. 2(a). The profile of average intensity across atomic columns lying parallel to $A B$ (along the $\{002\}$ planes) versus the perpendicular distance has been plotted in Fig. 2(b). The higher background intensity in the $\gamma$ matrix is due to the segregation of heavier alloying elements (such as $\mathrm{Cr}, \mathrm{Co}, \mathrm{W}, \mathrm{Nb}$ ). In addition to the expected superlattice contrast in the $\gamma^{\prime}$ phase and lack of it in the $\gamma$ phase, a definite transition zone is observed at the $\gamma / \gamma^{\prime}$ interface in Fig. 2(b). The ratio of the intensity of each atomic column to its adjacent column on the right can be used as a qualitative measure of the presence or absence of long-range chemical ordering, as shown in Fig. 2(c). Thus, for the $\gamma$ phase this ratio remains essentially constant at a value near 1 , since the site occupancies on the $\mathrm{Ni}$ and $\mathrm{Al}$ sublattices is equal in a random solid solution, while for
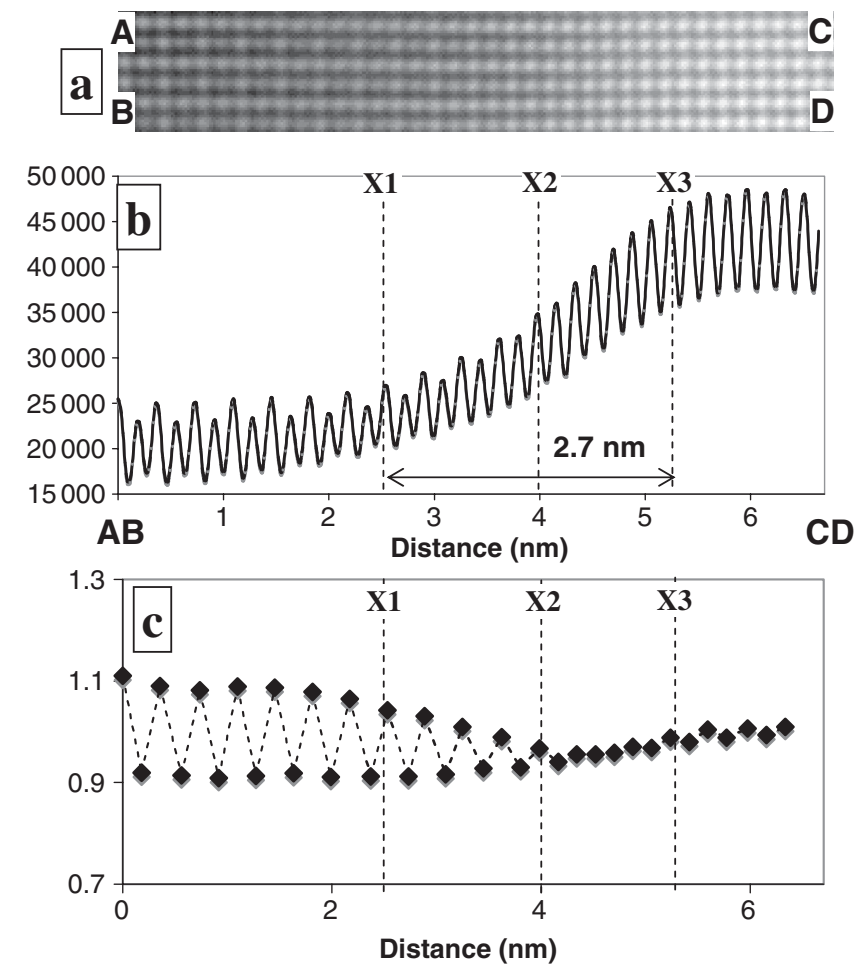

FIG. 2. (a) HRSTEM image from box $A B C D$ delineated in Fig. 1(c). (b) Averaged intensity profiles across row AB through CD showing the transition from ordered $\gamma^{\prime}$ to disordered $\gamma$. (c) Progressive intensity ratios showing the order-disorder transition and compositional width of the interface. 
the ordered $\gamma^{\prime}$ phase the ratio alternates between $\sim 1.1$ and 0.9 due to the differences in the site occupancies. In Fig. 2(c) a clear order-disorder transition zone can be defined at the $\gamma / \gamma^{\prime}$ interface $(X 1-X 2)$ where the long-range order decreases roughly over $6-8$ atomic $\{002\}$ planes (approximately 1 to $1.3 \mathrm{~nm}$ ). The same transition zone has also been marked at the corresponding location in Fig. 2(b). Similarly, based on the averaged intensities of the $\{002\}$ planes [Fig. 2(a)], a compositional transition zone (X1-X3 in Fig. 2(b), measuring $2.7 \mathrm{~nm}$ ), from the lower intensity columns (lower $Z$ ) on the $\gamma^{\prime}$ side, to the higher intensity columns (higher $Z$ ) on the $\gamma$ side, can now be defined. It is important to note that the $X 1-X 2$ orderdisorder transition zone lies completely within the $X 1-X 3$ compositional gradient, while the $X 2-X 3$ zone, where averaged intensity ratios progressively tend towards 1 , corresponds to a purely compositional transition within the $\gamma$ phase, discussed in more detail below. Measurements of the interface width, as determined through HRSTEM studies, were made by calculating the average and standard deviation of the peak intensities representing the $\mathrm{Ni}$ and $(\mathrm{Ni}+\mathrm{Al} / \mathrm{Ti})$ planes, respectively, in the $\gamma^{\prime}$, and the intensity peaks in the $\gamma$ phase. The interface width for each measurement was defined as the minimum interval (in integral $\{002\}$ plane spacings) between the $95 \%$ CI values separating the $\gamma$ and $\gamma^{\prime}$. An average value of approximately $2.2 \mathrm{~nm}$ (between 12-14 \{002\} planes), with a standard deviation of approximately $0.5 \mathrm{~nm}$, was obtained over several measurements of the interface width. Note that due to the small lattice mismatch between the $\gamma$ matrix and $\gamma^{\prime}$ precipitates in this alloy $(<0.05 \%)$, the elastic strains at the interface are small; consequently, any thinfoil relaxation effects on the experimentally observed compositional gradients, are expected to be relatively low.

The above HRSTEM studies have been complemented with 3DAP experiments for quantitatively probing the compositional gradients across the $\gamma / \gamma^{\prime}$ interface. Needle-shaped 3DAP specimens with a tip radius $\sim 50 \mathrm{~nm}$ were prepared (from the same Rene' 88 DT sample) by a combination of electrodischarge machining (EDM), electropolishing, and focused ion beam (FIB) techniques [32]. 3DAP experiments were conducted on a LEAP $^{\mathrm{TM}} 3000$ microscope manufactured by Imago Scientific Instruments, Inc. Figure 3(a) shows a 3D reconstruction depicting isoconcentration surfaces for Co (19 at \%), corresponding to the $\gamma$, and $\mathrm{Al}$ (10 at \%), corresponding to the $\gamma^{\prime}$. From the size scale, it is apparent that these are sections of primary $\gamma^{\prime}$ precipitates separated by a $\gamma$ channel. A 3DAP reconstruction of only $\mathrm{Al}$ atoms (black dots), exhibiting the higher concentration of $\mathrm{Al}$ within the $\gamma^{\prime}$ phase, is shown in Fig. 3(b). Figure 3(c) is a magnified view of the lower $\gamma / \gamma^{\prime}$ interface from Fig. 3(b), showing ordered planes of $\mathrm{Al}$ atoms with a plane spacing of $\sim 0.36 \mathrm{~nm}$, corresponding to the alternate Al-rich $\{002\}$ planes in the ordered $\gamma^{\prime}$ phase, confirming that the $\gamma / \gamma^{\prime}$
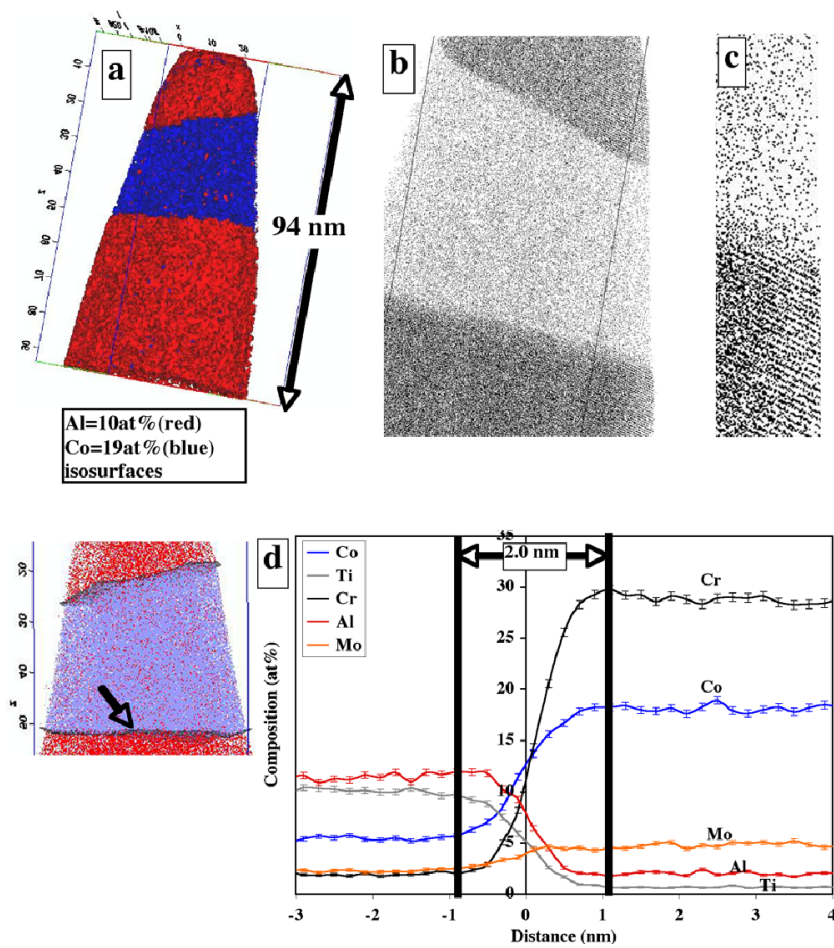

FIG. 3 (color online). (a) Al 10\% (red) and Co 19\% (blue) isosurfaces depicting morphology of the $\gamma^{\prime}$ precipitate used in 3DAP analysis; the red area is indicative of the primary $\gamma^{\prime}$ precipitate, (b) and (c) higher magnification views showing the stacking of alternate (002) Al planes within the $\gamma^{\prime}$. (d) Proximity histogram depicting the compositional transition across the $\gamma / \gamma^{\prime}$ interface; the area used in the analysis is indicated by the arrow in the inset.

interface is perpendicular to the $(z)$ axis of the sample. Note that Fig. 3(c) exhibits an order-disorder transition zone $\sim 4-6\{002\}$ planes wide $(0.7-1 \mathrm{~nm})$, in good agreement with the HAADF-HRSTEM results. For a statistically relevant quantitative analysis of the compositional gradient across this interface [indicated by an arrow in Fig. 3(d)], compositional profiles for the primary alloying elements have been plotted in the form of a proximity histogram [33] in Fig. 3(d). While heavier $\mathrm{Cr}$ and $\mathrm{Co}$ partition preferentially to the $\gamma$, the lighter $\mathrm{Al}$ and $\mathrm{Ti}$ partition to the $\gamma^{\prime}$ with a clear gradient across the interface, consistent with the HRSTEM observations shown in Figs. 2(b) and 2(c). Based on the $\mathrm{Cr}$ and Co profiles, the width of the compositional gradient across the interface shown can be measured to be $\sim 2.0 \mathrm{~nm}$ (average value $\sim 2.4 \mathrm{~nm}$ ), in excellent agreement with the HAADFHRSTEM observations.

Recent results on atomistic modeling of the $\gamma / \gamma^{\prime}$ interface in the binary Ni-Al system by Mishin [9] and Ardell and Ozolins [13] predict that the order-disorder interface is not atomically abrupt. Mishin's results on $\mathrm{Ni} / \mathrm{Ni}_{3} \mathrm{Al}$ interfaces [9] indicate that the decay in long-range order across the interface (measured through variation in the $\mathrm{Al}$ - 
sublattice occupancy across the interface) can occur over $4-6$ atomic layers or $\sim 0.7-1.0 \mathrm{~nm}$. This predicted value is in excellent agreement with our experimentally observed width of the order-disorder transition zone at the interface. Furthermore, the HAADF-HRSTEM and 3DAP observations indicate significant tolerance to compositional offstoichiometry within the $\gamma^{\prime}$ phase. Referring to Fig. 2(b), the transition zone from $\gamma^{\prime}$ to $\gamma$ is largely accounted for by a progressive increase in the intensity of alternate atomic columns belonging to the mixed $\mathrm{Al}+\mathrm{Ni}$ sublattices, attributable to decreasing $\mathrm{Al}$ occupancy (and, consequently, increasing $\mathrm{Ni}$ or other elemental occupancy), in excellent agreement with the predictions of atomistic simulations of the binary Ni-Al system [9]. It is important to note that these simulations, performed on binary systems, may not directly predict the existence of the two experimentally observed interface widths.

Coupling of HAADF-HRSTEM $Z$ contrast atomic resolution imaging with 3DAP Tomography serves to illustrate that the order-disorder interface in a nickel base alloy is not atomically abrupt but has a transition width, in agreement with prior atomistic simulations $[9,13]$. Furthermore, two transition "zones" can be defined, with the width of the order-disorder transition zone being smaller than the width of the compositional gradient. These results thus raise fundamental questions regarding the basic definition of an interface between an ordered phase and the surrounding disordered matrix and in general between two structurally and compositionally distinct phases. The structural and compositional diffuseness of the interface impacts the microstructural stability and mechanical properties of these metallic alloys, as it influences key parameters such as lattice misfit and coherency strains, which in turn directly impact the strengthening mechanisms and deformation behavior at elevated temperatures. The atomic-scale knowledge of structure and chemical composition is essential for a fundamental physically based understanding of such complex interfaces.

This work was funded in part by the Air Force Office of Scientific Research under the management of Dr. Joan Fuller and the Air Force Research Laboratory (AFRL). The support and facilities from the Center for the Accelerated Maturation of Materials (CAMM) at The Ohio State University and the Center for Advanced Research and Technology (CART) at the University of North Texas are gratefully acknowledged.

[1] H. Yamada et al., Science 305, 646 (2004).

[2] A. Ziegler et al., Science 306, 1768 (2004).

[3] D. A. Muller et al., Nature (London) 399, 758 (1999).
[4] C. L. Jia, M. Lentzen, and K. Urban, Science 299, 870 (2003).

[5] C. L. Jia and K. Urban, Science 303, 2001 (2004).

[6] H. Muller, S. Uhlemann, P. Hartel, and M. Haider, Microsc. Microanal. 12, 442 (2006).

[7] M. Haider, S. Uhlemann, and J. Zach, Ultramicroscopy 81, 163 (2000).

[8] H. Rose, Ultramicroscopy 103, 1 (2005).

[9] Y. Mishin, Acta Mater. 52, 1451 (2004).

[10] T. Kitashima, T. Yokokawa, A. C. Yeh, and H. Harada, Intermetallics 16, 779 (2008).

[11] I. M. Lifshitz and V. V. Slyozov, J. Phys. Chem. Solids 19, 35 (1961).

[12] C. Wagner, Z. Elektrochem. 65, 581 (1961).

[13] A. J. Ardell and V. Ozolins, Nature Mater. 4, 309 (2005).

[14] D. D. Krueger, R. D. Kissinger, R. D. Menzies, and C. S. Wukusick, U.S. Patent 49575671990.

[15] D. D. Krueger, R. D. Kissinger, R. D. Menzies, in Superalloys 1992, edited by S. D. Antolovich et al. (TMS-AIME, Warrendale, PA, 1992), p. 277.

[16] S. T. Wlodek, M. Kelly, D. A. Alden, in Superalloys 1996, edited by R. D. Kissinger et al. (TMS, Warrendale, PA, 1996), p. 129.

[17] G. B. Viswanathan et al., Acta Mater. 53, 3041 (2005).

[18] J. Telesman et al., in Superalloys 2004, edited by K. A. Green et al. (TMS Publications, Warrendale, PA, 2004), p. 215.

[19] N. S. Stoloff, in Superalloys II, edited by C. T. Sims, N. S. Stoloff, and W. C. Hagel (John Wiley and Sons, New York, NY, 1987), p. 61.

[20] K.E. Yoon, R.D. Noebe, O.C. Hellman, and D. N. Seidman, Surf. Interface Anal. 36, 594 (2004).

[21] M. K. Miller, Micron 32, 757 (2001).

[22] K. E. Yoon, D. Isheim, R. D. Noebe, and D. N. Seidman, Interface Sci. 9, 249 (2001).

[23] D. Blavette, E. Cadel, and B. Deconihout, Mater. Charact. 44, 133 (2000).

[24] D. Blavette, A. Bostel, and J. M. Sarrau, Metall. Trans. A 16, 1703 (1985).

[25] A. Buchon, S. Chambreland, and D. Blavette, Acta Conf. Du Coll. Nat. Sup. Monoc., Nancy 129 (1990).

[26] D. Blavette, L. Letellier, A. Racine, and A. Hazotte, Microsc., Microanal., Microstruct. 7, 185 (1996).

[27] P. D. Nellist and S. J. Pennycook, Science 274, 413 (1996).

[28] R. F. Klie, Y. Ito, S. Stemmer, and N. D. Browning, Ultramicroscopy 86, 289 (2001).

[29] R. F. Klie and Y. Zhu, Micron 36, 219 (2005).

[30] Defense Advanced Research Projects Agency, Defense Sciences Office (DSO), Engine Systems Prognosis, Contract No. HR0011-04-C-0001, HR0011-04-C-0002.

[31] P. M. Sarosi, G. B. Viswanathan, D. Whitis, and M. J. Mills, Ultramicroscopy 103, 83 (2005).

[32] J. Y. Hwang et al., Metall. Mater. Trans A 40, 24 (2009).

[33] O.C. Hellman et al., Microsc. Microanal. 6, 437 (2000). 